\title{
Visual field characteristics in neuromyelitis optica in absence of and after one episode of optic neuritis
}

This article was published in the following Dove Press journal:

Clinical Ophthalmology

10 June 2013

Number of times this article has been viewed

\author{
Harold Merle' \\ Stéphane Olindo ${ }^{2}$ \\ Séverine Jeannin ${ }^{2}$ \\ Rabih Hage' \\ Angélique Donnio' \\ Raymond Richer' \\ Philippe Cabre ${ }^{2}$ \\ 'Service d'Ophtalmologie, ${ }^{2}$ Service \\ de Neurologie, Centre Hospitalier \\ Universitaire de Fort de France, Fort- \\ de-France, Martinique, France
}

Purpose: Optic neuritis (ON) observed during neuromyelitis optica (NMO) is in most cases very severe and with poor prognosis. This study's objective was to analyze visual field (VF) abnormalities observed in the absence of $\mathrm{ON}$ and post-ON episode.

Methods: Twenty-seven cases of both NMO and multiple sclerosis (MS) were selected. Thorough ophthalmologic exam was performed at least 6 months post-ON attack. The VF was collected using the Humphrey 750 perimeter. We used the central threshold tests 24-2 with FASTPAC strategy. The abnormalities were categorized based on the Optic Neuritis Treatment Trial classification.

Results: After one ON, 40\% of the NMO group's eyes showed total VF loss $(P=0.01), 21 \%$ showed abnormalities of neurologic aspect, and $27 \%$ showed fascicular abnormalities of which $12 \%$ were altitudinal. Given the total VF loss, the positive predictive value in favor of an NMO was $92.8 \%$ and the negative predictive value was $47.3 \%$.

Conclusion: Alterations of the VF during the NMO differ from those observed in the course of the MS. One ON, blinding from the first attack, must call to mind an NMO. The altitudinal deficits point to a vascular mechanism.

Keywords: optic neuritis, visual field, neuromyelitis optica, multiple sclerosis

\section{Introduction}

Neuromyelitis optica (NMO), a rare inflammatory and demyelinating disease, impacts specifically the optic nerves and spinal cord. ${ }^{1,2}$ This pathology is predominantly found among women (80\%), spread worldwide, and has poor prognosis. Many clinical, immunological, and histological characteristics separate NMO from multiple sclerosis (MS), including sparing of the brain at the onset of the disease, frequent association $(10 \%-40 \%)$ with autoimmune diseases, diffuse damage of the white and grey matter of the spinal cord, as well as the involvement of functional and vital prognosis in the short term. ${ }^{3}$

Of unknown etiology, NMO is now considered an autoimmune disease linked to humoral immunity pathology, as recently determined based on the finding of the antibody directed against aquaporin 4 (AQP4-Ab). ${ }^{4}$ Lesions are characterized by perivascular immunoglobulin (IgM) and complement (C9) deposits, but also by necrosis zones. ${ }^{5}$

Visual function is severely affected as confirmed by frequent ocular attacks and severity of its degradation. ${ }^{6}$ A remote optic neuritis (ON), prior to NMO, is often considered idiopathic or due to MS; the most frequent treatment is intravenous corticotherapy with high dosage, which generally has poor results. ${ }^{7}$ Recently, plasma
Hôpital Pierre Zobda-Quitman, BP 632

9726I Fort de France Cedex, Martinique, France (French West Indies)

Tel +33596596552 252

Fax +33 596596758447

Email harold.merle@chu-fortdefrance.fr 
exchanges have been shown to be very efficient treatments for $\mathrm{ON}$ due to $\mathrm{NMO}$, increasing the need to differentiate $\mathrm{ON}$ due to NMO as soon as possible from ON due to MS. ${ }^{8-10}$

The study's objective was to analyze visual field (VF) abnormalities observed in the absence of ON and post-ON episode as well as to specify their role in early NMO diagnosis.

\section{Methods}

Our study was conducted at the University Hospital Center of Fort de France in Martinique, where all patients have been regularly followed since 1985 . All patients had no previous history of any of the following ON: familial, infectious, glaucoma related, vascular, compressive, due to general diseases, toxic, or due to deficiency. All patients were human immunodeficiency virus (HIV) and human T-lymphotropic virus 1 (HTLV-1) negative.

\section{Population studied}

Twenty-seven NMO cases were selected. The NMO diagnosis was based on Wingerchuk et al's diagnostic criteria established in 1999 and revised in 2006. ${ }^{11,12}$ The revised diagnostic criteria require the presence of two absolute criteria as well as of two out of three major criteria. The absolute criteria are: the existence of unified or bilateral ON, and of acute myelitis. The major criteria are: normal magnetic resonance imaging (MRI) at the onset of the disease, hypersignal extended to at least three vertebral segments on the medullar MRI, and positive search results for AQP4-Ab. The acute myelitis diagnosis was based on the presence of medullar damage including sphincter, sensitive, or motor disorders, for which maximum deficit is reached in less than 4 weeks. Ocular or medullar attack was defined as the apparition or worsening of signs or symptoms for at least 24 hours. A second attack was a new event that happened at least 1 month after the initial attack. The final index attack confirmed the diagnosis. We revealed that the optic-medullary period matched the time frame that separated the first and final index attack. The disease's global handicap was ranked using the Kürtzke scale from 0 to 10 (expanded disability status scale [EDSS]). ${ }^{13}$ We calculated the handicap progression rapport as followed: EDSS/length of the disease (years).

We compared the results of the NMO patients with those obtained in the MS patients' group matched by age and sex. The MS patients $(\mathrm{n}=27)$ met McDonald et al's diagnosis criteria. $^{14}$

We used our Martinique NMO and MS patients' database to obtain characteristics of the disease's history. Data was collected prospectively over a 20 -year period. All patients were tested for AQP4-Ab.

\section{Analysis}

For each patient, a thorough eye exam was conducted at least 6 months post-ON onset, including a precise measure of refraction. Visual acuity was collected using the Snellen and Early Treatment Diabetic Retinopathy Study (ETDRS) scales. Sensibility to spatial contrast was measured with the Pelli-Robson and Sloan charts. ${ }^{15}$ On the Pelli-Robson test, letters are organized by groups of triplets of different contrast. ${ }^{11}$ An identified triplet consists of two out of the three letters being correctly recognized. A score of 15 or higher (converted in logarithmic units for statistical analysis) is considered normal. According to Sloan, weak contrast cards depend on the identification of grey letters, whose size progressively decreases. The format is similar to the ETDRS scale, and has five letters per line. These letters appear on a backlit white base (Precision Vision, La Salle, IL, USA) and are tested at $2 \mathrm{~m}$. We used two levels of contrast $(1.25 \%$ and $2.5 \%$ ). Each letter read correctly contributed one point to the total score. The total score was the addition of all correctly read letters (0-70). We assessed colors vision with the 100-hue test of Farnsworth. The square root of the score was used for statistical analysis. The score's normal value by age was obtained using Verriest's tables. We also used frequency doubling technology perimetry (FDTP) (Carl Zeiss Meditec AG, Jena, Germany). We used the N-30 threshold program. The thickness of retinal peripapillary nerve fibers was measured with a Stratus OCT (Carl Zeiss Meditec AG) equipped with the 4.0 version of the software. We followed the fast retinal nerve fiber layer (RFNL) thickness protocol to collect data. The equipment produced three circular scans (resolution: 256) of $3.4 \mathrm{~mm}$ of diameter centered on the head of the optic nerve in $1.92 \mathrm{~s}$. We measured the average thickness of the layer of retinal nerve fiber, as well as the average thickness in the temporal, superior, nasal, and inferior quadrants. The quality of input of selected scans was superior or equal to seven. In the case of a narrow pupil, the OCT was realized after instillation of a drop of tropicamide.

Standard automated perimetry (SAP) was done using the Humphrey Field Analyzer 750 II (Carl Zeiss Meditec AG). We used the test of central threshold 24-2 (54 points tested) with FASTPAC strategy. The VF was considered reliable if false positives, false negatives, and fixation losses were below $33 \%$. A VF was definitely normal if all locations were within normal limits on the total deviation plot. A VF was definitely abnormal if any of the following conditions were met: (1) the Glaucoma Hemifield Test VF index was 
Table I Classification of the visual field abnormalities

I. Neurologic abnormalities: chiasmal, retrochiasmal, optic nerve chiasm

I.I. Vertical step

Limited visual field loss that respects the vertical meridian and that includes at least two abnormal points at or outside $15^{\circ}$ along the vertical meridian.

I.2. Quadrant Significant visual field loss throughout an entire quadrant that respects the vertical midline. Essentially all points must have a $P<5 \%$ value on the total deviation plot.

I.3. Partial hemianopia

A visual field defect that respects the vertical meridian and that is greater than one quadrant but less than a complete vertical hemifield.

I.4. Hemianopia

A visual field defect that respects the vertical meridian and that involves essentially all points in a vertical hemifield.

I.5. Three quadrant

Significant visual field loss throughout three quadrants; essentially all points must have a $P<5 \%$ value on the total deviation plot.

\section{Optic nerve abnormalities}

II.I. Nerve fiber bundle abnormalities

II.I.a. Temporal wedge

II.I.b. Enlarged blind spot

II.I.c. Nasal step

II.I.d. Paracentral

II. I.e. Partial arcuate

II.I.f. Arcuate

II.I.g. Altidudinal

II.2. Diffuse abnormalities II.2.a. Multiple foci

II.2.b. Widespread

II.3. Central abnormalities II.3.a. Centrocecal

II.3.b. Central

II.4. Severe abnormalities II.4.a. Total loss of vision

III. Artifactual abnormalities
A small visual field defect that is temporal to the blind spot.

A visual field abnormality in the nerve fiber bundle region that involves at least two points and is contiguous with the blind spot.

Limited visual field loss adjacent to the nasal horizontal meridian with at least one abnormal point at or outside $15^{\circ}$ on the meridian. Cannot include more than one significant point (on either plot) in the nerve fiber bundle region on the temporal side.

A relatively small visual field abnormality in the nerve fiber bundle region that is generally not contiguous with the blind spot or the nasal meridian. In particular, it does not involve points outside $15^{\circ}$ that are adjacent to the nasal meridian.

Visual field loss in the nerve fiber bundle region that extends incompletely from the blind spot to the nasal meridian. The defect is generally contiguous with either the blind spot or the nasal meridian and must include at least one abnormal location in the temporal visual field.

Significant visual field loss in the nerve fiber bundle region, extending across contiguous abnormal points from the blind spot to at least one point outside $15^{\circ}$ adjacent to the nasal meridian.

Severe visual field loss throughout the entire superior or inferior hemifield that respects the horizontal midline, with most points in the hemifield having a $P<5 \%$ value on the total deviation plot and the entire horizontal midline demonstrating abnormality.

Visual field loss that includes two or more clusters of abnormal points $(P<5 \%)$ located in different areas of the visual field that do not correspond to a particular abnormality pattern. The loss must be present in both the total and pattern deviation plots.

Diffuse visual field loss that includes all four quadrants. The Glaucoma Hemifield Test may show a general reduction of sensitivity or the MD must have a $P$ level $<5 \%$. The corrected pattern standard deviation must not have a $P$ level $<5 \%$. Most abnormal points on the total deviation plot are not abnormal on the pattern deviation plot.

Visual field loss that is in the macular region and contiguous with the blind spot. The foveal threshold must have a $P<5 \%$ value. The loss must be symmetrical above and below the midline.

Visual field loss that is predominantly in the macular region. The foveal threshold must have a $P<5 \%$ value, and the visual acuity must be impaired. Can be associated with a single hemifield and paired with another defect.

Severe widespread visual field loss (MD $\leq-20.00 \mathrm{~dB}$ ) with visual acuity not totally impaired.

I. Superior depression: Two or more abnormal points in the very superior region.

2. Inferior depression: Two or more abnormal points in the very inferior region.

3. Partial peripheral rim: Generally continuous visual field loss outside $15^{\circ}$, but not in all quadrants; must have some curvature.

4. Peripheral rim: Generally continuous visual field loss outside $15^{\circ}$ in all four quadrants, usually with no visual field loss inside $15^{\circ}$ on either deviation plot. There must be visual field loss temporal to the blind spot.

Abbreviation: MD, mean deviation.

abnormal; (2) the corrected pattern standard deviation VF index was abnormal $(P<5 \%)$; (3) a single point was worse than the $0.5 \%$ probability level on the total and/or pattern deviation plot; (4) two clustered points are beyond the reference range $(P<5 \%)$, and at least one point was worse than the $P<1 \%$ on the total and/or pattern deviation plot (a cluster was defined as $\geq$ two horizontally or vertically, not diagonally, contiguous abnormal points with $P<5 \%$ ); and/or (5) three or more clustered points were worse than $P<5 \%$ on the total and/or pattern deviation plot. 
In order to determine the abnormality classification for the entire VF, we used the most predominant defect. The pattern of abnormal points on the deviation plot (total or pattern) showing the greater number of abnormal points, was used to determine the appropriate abnormality classification. When necessary, Goldmann's VF was also conducted in order to specify the deficit's form.

The VF abnormalities were ranked in 21 categories based on the Optic Neuritis Treatment Trial (ONTT) classification and reorganized into three principal groups: neurologic, optic nerve, and artifactual abnormalities (Table 1). ${ }^{16}$

The data was computerized and analyzed in a strictly anonymous manner using MS Excel (Microsoft Corporation, Redmond, WA, USA) and Statview (SAS Institute Inc, Cary, NC, USA). The following tests were used for statistical analysis: Chi-square test for frequency comparisons, Chi-square Yates correction for small populations, and Student's $t$-test for average comparisons. This study was approved by the Consulting Committee for the Protection of Individuals of Biomedical Research sponsored by the French Ministry of Health.

\section{Results}

Demographic characteristics of the two groups are displayed in Table 2. NMO patients were on average 47 years old and $92 \%$ were females. The average age at the NMO onset was $37.1 \pm 12.0$ years. NMO and MS had been evolving for at least 10 years. The optic-medullary period was $25.3 \pm 38.9$ months. $55 \%$ of the patients were positive to AQP4-Ab. There was no difference between the two groups regarding average age, sex ratio, age of onset, and length of

Table 2 Demographic comparison between the NMO and MS groups

\begin{tabular}{llll}
\hline & $\begin{array}{l}\text { NMO } \\
(\mathbf{n}=\mathbf{2 7})\end{array}$ & $\begin{array}{l}\text { MS } \\
(\mathbf{n}=\mathbf{2 7})\end{array}$ & $\begin{array}{l}\text { P-value } \\
\end{array}$ \\
\hline Age (years) & $47.5 \pm 10.5$ & $44.5 \pm 10.1$ & 0.2 \\
Sex ratio F/M (\%F) & $25 / 2(92.6 \%)$ & $22 / 5(81.5 \%)$ & 0.4 \\
Age at onset (years) & $37.1 \pm 12$ & $30.7 \pm 9.9$ & 0.053 \\
Disease duration (years) & $9.9 \pm 7.3$ & $11.7 \pm 8$ & 0.3 \\
Optic-medullary time & $25.3 \pm 38.9$ & - & \\
elapsed (years) & & & \\
AQP4-Ab positive & $15(55.5 \%)$ & 0 & \\
EDSS & $5.3 \pm 2.5$ & $3.85 \pm 2.17$ & 0.1 \\
IP EDSS & $0.95 \pm 1.51$ & $0.8 \pm 1.2$ & 0.2 \\
Ocular presenting symptom & $16(59.2 \%)$ & $8(29.6 \%)$ & 0.055 \\
Number of attacks/patient & $1.55 \pm 1.8$ & $0.63 \pm 1.07$ & 0.0002 \\
Number of attacks/patient & $1.4 \pm 0.84$ & $0.55 \pm 0.69$ & 0.0002 \\
in the first 2 years & & & \\
Annual relapsing rate & $0.44 \pm 0.8$ & $0.13 \pm 0.14$ & 0.0006 \\
\hline
\end{tabular}

Abbreviations: EDSS, expanded disability status scale; F, female; IP, index of progression; M, male; MS, multiple sclerosis; NMO, neuromyelitis optica. the disease. The number of attacks per patient, number of attacks the first 2 years, and the yearly rate of attacks were all higher in the NMO group.

Ten NMO and $30 \mathrm{MS}$ eyes had no previous history of ON. Thirty-three NMO and 19 MS eyes had one previous ON attack. Eight NMO and three MS eyes had two previous attacks. Four NMO and two MS eyes had more than two previous attacks.

The results of the visual function and of optic nerve fibers thickness in absence of and post-ON episode are presented in Table 3. For the eyes with no ON history, there was no difference in visual acuity (Snellen) and optic nerve fibers thickness between the two groups. Nevertheless, ETDRS visual acuity, contrasts vision (Sloan chart 1.25\%), mean deviation SAP, and foveal threshold were not as good in the MS group.

After one ON attack, Snellen visual acuity, ETDRS, and the optic nerve fibers thickness were smaller in the NMO group than in the MS group (20/50 versus 20/30, $P=0.02 ; 31.4$ versus $44.1, P=0.04 ; 64.4 \mu \mathrm{m}$ versus $77 \mu \mathrm{m}$, $P=0.06$, respectively). The proportion of eyes with a visual acuity $\leq 20 / 200$ was $42 \%$ in the NMO group versus $21 \%$ in the MS group $(P=0.03)$.

Table 4 shows the results of the VF morphological analysis in absence of and post-ON episode. In the absence of ON, the foveal threshold was 34 for the NMO group and 32.5 for the MS group $(P=0.07)$. Half of the NMO patients' VFs were normal, a third of the MS patients' VFs were normal, and one third of the MS patients'VFs showed diffuse lesions. In the absence of $\mathrm{ON}$ there was no difference in the distribution of VF deficits between the two groups.

Post-ON attack, the foveal threshold was 16.2 in the NMO group and 27.4 in the MS group $(P=0.01)$, and almost $40 \%$ of the NMO group's eyes showed total VF loss versus only $5.3 \%$ for MS group's eyes $(P=0.01)$. In the case of total VF loss, the positive predictive value in favor of NMO was $92.8 \%$ and the negative predictive value was $47.3 \%$. In the NMO group, neurological abnormalities were found in $21.3 \%$ of cases and fascicular abnormalities in $27.3 \%$, of which $12.1 \%$ were altitudinal deficits (Figures 1 and 2). No altitudinal deficit was observed in the MS group. Diffuse abnormalities were found in $42.1 \%$ of the MS eyes versus $6 \%$ of the NMO eyes $(P=0.005)$. In the presence of a diffuse deficit, the positive predictive value in favor of MS was $80 \%$ and the negative predictive value $73.8 \%$.

\section{Discussion}

Our study showed that after a first ON attack, $40 \%$ of the NMO patients' eyes presented total VF loss and that the ON 
Table 3 Visual function and optic nerve fibers thickness in the absence of prior history and after an optic neuritis episode

\begin{tabular}{|c|c|c|c|c|c|c|}
\hline \multirow{2}{*}{$\begin{array}{l}\text { Number of optic neuritis } \\
\text { episodes }\end{array}$} & \multicolumn{3}{|l|}{0} & \multicolumn{3}{|l|}{ I } \\
\hline & $\begin{array}{l}\text { NMO } \\
(n=10 \text { eyes })\end{array}$ & $\begin{array}{l}\text { MS } \\
(n=30 \text { eyes })\end{array}$ & $P$-value & $\begin{array}{l}\text { NMO } \\
(n=33 \text { eyes })\end{array}$ & $\begin{array}{l}\text { MS } \\
(n=19 \text { eyes })\end{array}$ & $P$-value \\
\hline Visual acuity (Snellen) & $20 / 20$ & $20 / 20$ & 0.4 & $20 / 50$ & $20 / 30$ & 0.02 \\
\hline Visual acuity ETDRS & $61.1 \pm 4.6$ & $55.6 \pm 5.6$ & 0.006 & $31.4 \pm 24.6$ & $44.1 \pm 18.6$ & 0.04 \\
\hline Visual acuity $\leq 20 / 200$ & 0 & 0 & - & 14 (42.4\%) & $4(2 \mid \%)$ & 0.03 \\
\hline Pelli-Robson chart & $1.7 \pm 0.4$ & $1.6 \pm 0.1$ & 0.08 & $0.93 \pm 0.7$ & $1.2 \pm 0.5$ & 0.09 \\
\hline Sloan chart $1.25 \%$ & $20.9 \pm 7.1$ & $12.1 \pm 9.5$ & 0.02 & $3 \pm 5.9$ & $6 \pm 7.9$ & 0.3 \\
\hline Sloan chart $2.5 \%$ & $30.4 \pm 11.2$ & $26.2 \pm 9.9$ & 0.06 & $9.3 \pm 13.3$ & $15 \pm 14$ & 0.1 \\
\hline MD (SAP) & $-1.8 \pm 0.9$ & $-3.7 \pm 3.1$ & 0.01 & $-10.3 \pm 10$ & $-7.9 \pm 5.7$ & 0.9 \\
\hline PSD (SAP) & $2 \pm 0.4$ & $2.9 \pm 1.4$ & 0.007 & $5 \pm 3.1$ & $4.8 \pm 2.5$ & 0.9 \\
\hline Foveal threshold & $34.4 \pm 1.8$ & $32.5 \pm 3.1$ & 0.07 & $16.2 \pm 15.2$ & $27.4 \pm 10.4$ & 0.01 \\
\hline MD (FDTP) & $-0.5 \pm 3.3$ & $-3.1 \pm 3.8$ & 0.09 & $-6.2 \pm 5.9$ & $-3.4 \pm 3.6$ & 0.1 \\
\hline PSD (FDTP) & $3.5 \pm 0.4$ & $5.6 \pm 2.8$ & 0.01 & $6.7 \pm 3.5$ & $6.2 \pm 3.3$ & 0.3 \\
\hline 100-Hue & $43.7 \pm 53.4$ & $74.1 \pm 91$ & 0.4 & $91.4 \pm 91.2$ & $135.5 \pm 142.3$ & 0.3 \\
\hline $\begin{array}{l}\text { Overall average RNFL } \\
\text { thickness }(\mu \mathrm{m})\end{array}$ & $95.6 \pm 12.1$ & $96.3 \pm 13$ & 0.5 & $64.4 \pm 22.8$ & $77 \pm 21.2$ & 0.06 \\
\hline
\end{tabular}

Abbreviations: ETDRS, Early Treatment Diabetic Retinopathy Study; FDTP, frequency doubling technology perimetry; MD, mean deviation; MS, multiple sclerosis; NMO, neuromyelitis optica; PSD, pattern standard deviation; RNFL, retinal nerve fiber layer; SAP, standard automated perimetry.

complicated with total VF loss was, over nine out of ten times, an NMO. In Fernandes et al's study, after a single ON episode, a mean deviation lower than $-20 \mathrm{~dB}$ was associated with a 6.0 odds ratio for NMO.${ }^{17} \mathrm{In}$ two out of three cases in another study, NMO started with ON, sometimes bilaterally. ${ }^{6}$ It was rare to see the $\mathrm{ON}$ associated with myelitis or appearing after the discovery of medullar lesions. Lennon et $\mathrm{al}^{3}$ identified a biological marker (AQP4-Ab) to differentiate NMO from MS. However, this antibody directed against aquaporin-4 has a sensitivity of $60 \%-70 \%$ and specificity of $91 \% .{ }^{4,18}$

Table 4 Visual field abnormalities in absence of and after one optic neuritis episode

\begin{tabular}{|c|c|c|c|c|c|c|}
\hline \multirow{2}{*}{$\begin{array}{l}\text { Number of optic neuritis } \\
\text { episodes }\end{array}$} & \multicolumn{3}{|l|}{0} & \multicolumn{3}{|l|}{ I } \\
\hline & $\begin{array}{l}\text { NMO } \\
(n=10 \text { eyes })\end{array}$ & $\begin{array}{l}\text { MS } \\
(n=30 \text { eyes })\end{array}$ & $P$-value & $\begin{array}{l}\text { NMO } \\
(n=33 \text { eyes })\end{array}$ & $\begin{array}{l}\text { MS } \\
(n=19 \text { eyes })\end{array}$ & P-value \\
\hline Foveal threshold & $34 \pm 1.8$ & $32.5 \pm 3.1$ & 0.07 & $16.2 \pm 15.2$ & $27.4 \pm 10.4$ & 0.01 \\
\hline I. Neurologic abnormalities & 0 & I (3.3\%) & 0.9 & $7(2 \mathrm{I} .3 \%)$ & $3(15.8 \%)$ & 0.9 \\
\hline I.I. Vertical step & 0 & 0 & - & 0 & 0 & - \\
\hline I.2. Quadrant & 0 & 0 & - & $3(9 \%)$ & 0 & 0.4 \\
\hline I.3. Partial hemianopia & 0 & 0 & - & I (3\%) & 0 & 0.9 \\
\hline I.4. Hemianopia & 0 & 0 & - & I (3\%) & 0 & 0.9 \\
\hline I.5. Three quadrant & 0 & I (3.3\%) & 0.9 & $2(6 \%)$ & $3(15.8 \%)$ & 0.5 \\
\hline II.I. Nerve fiber bundle abnormalities & $3(30 \%)$ & $8(26.7 \%)$ & 0.9 & $9(27.3 \%)$ & $4(21 \%)$ & 0.9 \\
\hline II.I.a. Temporal wedge & 0 & 0 & - & 0 & 0 & - \\
\hline II.I.b. Enlarged blind spot & 0 & 0 & - & 0 & I (5.3\%) & 0.9 \\
\hline II.I.c. Nasal step & 0 & $3(10 \%)$ & 0.7 & I (3\%) & 0 & 0.9 \\
\hline II.I.d. Paracentral & $3(30 \%)$ & $4(13.3 \%)$ & 0.4 & $3(9 \%)$ & 0 & 0.4 \\
\hline II.I.e. Partial arcuate & 0 & 0 & - & 0 & I (5.3\%) & 0.9 \\
\hline II.I.f. Arcuate & 0 & I (3.3\%) & 0.9 & I (3\%) & $2(10.5 \%)$ & 0.6 \\
\hline II.I.g. Altidudinal & 0 & 0 & - & $4(12.1 \%)$ & 0 & 0.3 \\
\hline II.2. Diffuse abnormalities & $2(20 \%)$ & $9(30 \%)$ & 0.8 & $2(6 \%)$ & $8(42.1 \%)$ & 0.005 \\
\hline II.2.a. Multiple foci & $2(20 \%)$ & $4(13.3 \%)$ & 0.9 & 0 & $2(10.5 \%)$ & 0.2 \\
\hline Il.2.b. Widespread & 0 & $5(16.6 \%)$ & 0.4 & $2(6 \%)$ & $6(31.6 \%)$ & 0.04 \\
\hline II.3. Central abnormalities & 0 & $2(6.7 \%)$ & 0.9 & 0 & $2(10.5 \%)$ & 0.2 \\
\hline II.3.a. Centrocecal & 0 & 0 & - & 0 & I (5.3\%) & 0.9 \\
\hline Il.3.b. Central & 0 & $2(6.7 \%)$ & 0.9 & 0 & I (5.3\%) & 0.9 \\
\hline II.4.a. Total loss of vision & 0 & 0 & - & $13(39.4 \%)$ & I (5.3\%) & 0.01 \\
\hline Artifactual abnormalities & 0 & 0 & - & 0 & 0 & - \\
\hline Normal & $5(50 \%)$ & $10(33.3 \%)$ & 0.5 & $2(6 \%)$ & I (5.3\%) & 0.9 \\
\hline
\end{tabular}

Abbreviations: MS, multiple sclerosis; NMO, neuromyelitis optica. 

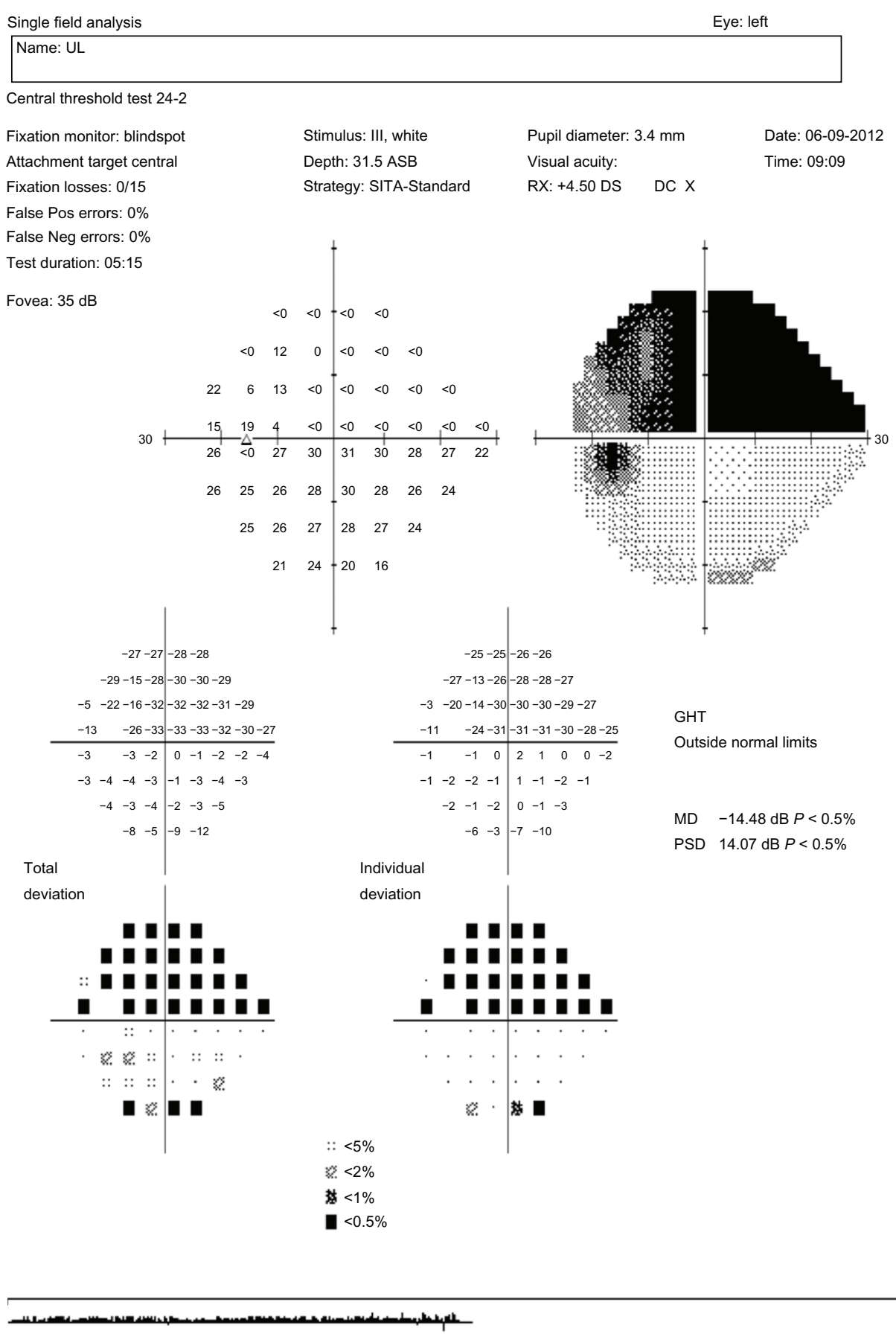

2005 Carl Zeiss Meditec

HFA II 750-11687-4.1/4.1

Figure I Standard automated perimetry.

Note: Left eye: altitudinal deficit.

Abbrevations: PSD, pattern standard deviation; MD, mean deviation; HFA, Humphrey Field Analyzer; DOB, date of birth; GHT, glaucoma hemifield test; RX, refraction; DC, power of the cylinder; Pos, positive; Neg, negative.

Further, in many cases, MRI of isolated ON is normal; ON is then considered idiopathic or due to MS. In contrast with the idiopathic or MS-related ON for which vision is usually recovered in a few weeks, ON due to NMO has a very poor prognosis. ${ }^{19}$
Thus, the relation to MS or NMO must be identified as soon as possible in order to start the proper treatment for the acute phase as well as a thorough immunosuppressant treatment. Besides MS, blinding ONs are exceptional. They only represent $3.6 \%$ of observed ON throughout the course of MS, even course 


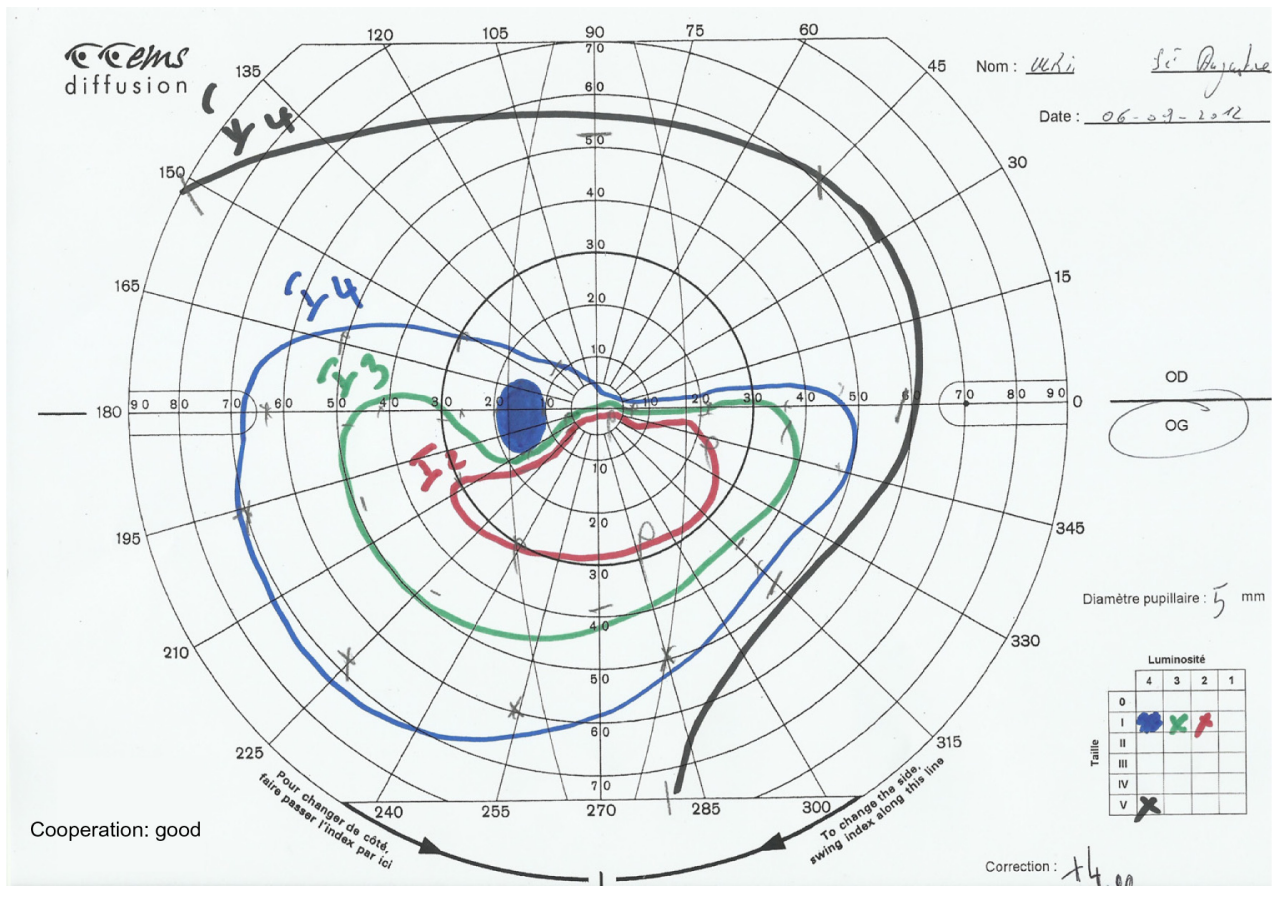

Figure 2 Goldmann's visual field. Note: Left eye: altitudinal deficit.

though $36.9 \%$ of patients show total vision loss at the acute phase. ${ }^{16}$ In Slamovits et al's study, among $12 \mathrm{ON}$ cases without light perception, eight recovered with a visual acuity superior to 20/40 and four with a visual acuity less than 20/40 but with periphery vision conservation. None stayed blind. ${ }^{20}$

A first $\mathrm{ON}$ attack complicated by total vision loss in an AQP4-Ab negative patient, female and of African, Indian, or Asian decent must be considered as the first NMO sign. Indeed, the ocular start and $\mathrm{ON}$ frequency are more frequent among these populations while the onset and frequency of medullar attacks is higher in the white population. ${ }^{21,22}$ Optical coherence tomography can also contribute to orient the NMO diagnosis when RNFL is inferior to $50 \mu \mathrm{m}$ and when mostly superior and inferior quadrants are concerned. ${ }^{23,24}$

Given the deficit's seriousness and fast onset of irreversible optic nerve lesions, the treatment of NMO due to $\mathrm{ON}$ is an emergency. Because of the good results obtained through plasma exchanges, we propose their systematic use in association with intravenous corticotherapy, rather than waiting to conclude the inefficacy of corticotherapy alone. . $^{8,10,25}$

Localized anomalies (neurologic anomalies and nerve fiber bundle anomalies) concern about $50 \%$ of the deficits in our series. Fernandes et al, whose series' numbers were similar to ours, also found half of the deficits to be localized. ${ }^{17}$ Nakajima et al collected VFs for 15 NMO patients with
Goldmann perimetry. ${ }^{26} \mathrm{He}$ found, as we did, about $25 \%$ of deficits with a neurological aspect. ${ }^{26}$ In Sadda et al's study, after a posterior ischemic $\mathrm{ON}$, one third of the eyes kept a visual acuity inferior to 20/640 and when measurable, 30\% of the VF showed altitudinal deficit. ${ }^{27}$ The rarity of diffuse lesions ( $6 \%$ in our series), the localized deficits depth, high proportion of neurological aspect deficits, as well as RNFL significant decrease of the four quadrants, show a severe axonal lesion compared to MS. These severe axonal lesions recall those observed throughout the course of vascular related neuropathies such as the ischemic or glaucomatous ON. ${ }^{23,24}$ In Fernandes et al's and our series, the proportion of localized deficits among MS patients was smaller, one third, and appropriate for the ONTT results at 1 year. ${ }^{17}$ Consequently, it is very common throughout the course of MS to observe a selective reach of the interpapillomacular bundle.

Four eyes (12.1\%) of NMO patients showed altitudinal deficits. This type of deficit was not found among the 27 eyes of Fernandes et al's series, but was noted as the most frequent of the non-central deficits in Nakajima et al's series. ${ }^{17,26}$ These altitudinal deficits had all been observed at the time of the first $\mathrm{ON}$ attack; they were superior in three cases and inferior in two cases. Altitudinal deficit is usually characteristic of ischemic ON linked to an occlusion of the posterior ciliary arteries. Encountered throughout the course of NMO, such a deficit brings to mind a vascular contribution 
in ON occurrence. Further, throughout the NMO, the optic nerve appears grayish and softened. The demyelinization predominates at the center of the nerve and is sometimes at the origin of a central cavitation. ${ }^{28}$ As a consequence of the AQP4-Ab presence, they are associated to perivascular immunoglobulin deposits (Ig M) and complement (C9), axonal necrosis lesions, and atrophy that are never observed throughout the MS. ${ }^{29}$ Other components in favor of the vascular contribution to $\mathrm{ON}$ hypothesis include the revelation of retinal vascular anomalies of attenuation type on the peripapillary arterioles and frosty or occluded aspect of the arterioles located at more than two papillary diameters of the papilla. ${ }^{30}$ All of these vascular anomalies would be the result of an inflammatory reaction caused by the AQP4-Ab directed against aquaporin-4, strongly expressed at the level of the optic nerve astrocytes and the arterioles of the retina's deep layer. ${ }^{31}$

Identifying NMO as soon as possible and putting in place the most adapted treatment is critical for a lesion with poor ocular prognosis and for which vital prognosis is very unfavorable. The VF lesions observed after one episode of $\mathrm{ON}$ in NMO are different from those usually observed in MS. Given a total VF loss, the positive predictive value in favor of an NMO was $92.8 \%$ and the negative predictive value was $47.3 \%$. Obtained with an ambispective study, these values should be confirmed with a prospective study. The VF analysis helps to invoke the NMO diagnosis when faced with severe or altitudinal lesions that appeared during the first $\mathrm{ON}$ attack.

\section{Acknowledgments}

We thank Agathe Merle, MPA, MPH (University of Wisconsin-Madison, Wisconsin), Karen Thérèse (CHU Fort de France), and Eric Ventura (CHU Fort de France).

\section{Disclosure}

The authors report no conflicts of interest in this work.

\section{References}

1. Devic E. Myélite subaiguë compliquée de névrite optique. Bull Méd. 1894;8:1033-1034.

2. Wingerchuk DM, Lennon VA, Lucchinetti CF, Pittock SJ, Weinshenker BG. The spectrum of neuromyelitis optica. Lancet Neurol. 2007;6(9): 805-815.

3. Lennon VA, Kryzer TJ, Pittock SJ, Verkman AS, Hinson SR. IgG marker of optic-spinal multiple sclerosis binds to the aquaporin-4 water channel. J Exp Med. 2005;202(4):473-477.

4. Lennon VA, Wingerchuk DM, Kryser TJ, et al. A serum autoantibody marker of neuromyelitis optica: distinction from multiple sclerosis. Lancet. 2004;364(9451):2106-2112.
5. Lucchinetti CF, Mandler RN, McGavern D, et al. A role for humoral mechanisms in the pathogenesis of Devic's neuromyelitis optica. Brain. 2002;125(Pt 7):1450-1461.

6. Merle H, Olindo S, Bonnan M, et al. Natural history of the visual impairment of relapsing neuromyelitis optica. Ophthalmology. 2007; 114(4):810-815.

7. Hickman SJ, Dalton CM, Miller DH, Plant GT. Management of acute optic neuritis. Lancet. 2002;360(9349):1953-1962.

8. Watanabe S, Nakashima I, Misu T, et al. Therapeutic efficacy of plasma exchange in NMO-IgG-positive patients with neuromyelitis optica. Mult Scler. 2007;13(1):128-132.

9. Yoshida H, Ando A, Sho K, et al. Anti-aquaporin-4 antibody-positive optic neuritis treated with double-filtration plasmapheresis. $J$ Ocul Pharmacol Ther. 2010;26(4):381-385.

10. Merle H, Olindo S, Jeannin S, et al. Treatment of optic neuritis by plasma exchange (add-on) in neuromyelitis optica. Arch Ophthalmol. 2012;130(7):858-862.

11. Wingerchuk DM, Hogancamp WF, O'Brien PC, Weinshenker BG. The clinical course of neuromyelitis optica (Devic's syndrome). Neurology. 1999;53(5):1107-1114.

12. Wingerchuk DM, Lennon VA, Pittock SJ, et al. Revised diagnostic criteria for neuromyelitis optica. Neurology. 2006;66(10):1485-1489.

13. Kurtzke JF. Rating neurologic impairment in multiple sclerosis: a expanded disability status scale (EDSS). Neurology. 1983;33(11): $1444-1452$.

14. McDonald WI, Compston A, Edan G, et al. Recommended diagnostic criteria for multiple sclerosis: guidelines from the International Panel on the diagnoses of multiples sclerosis. Ann Neurol. 2001;50(1): $121-127$.

15. Trobe JD, Beck RW, Moke PS, Cleary PA. Contrast sensitivity and other vision tests in the optic neuritis treatment trial. Am J Ophthalmol. 1996;121(5):547-553.

16. Keltner JL, Johnson CA, Cello KE, Dontchev M, Gal RL, Beck RW; Optic Neuritis Study Group. Visual field profile of optic neuritis. A final follow-up report from the optic neuritis treatment trial from baseline through 15 years. Arch Ophthalmol. 2010;128(3):330-337.

17. Fernandes DB, Ramos Rde I, Falcochio C, Apóstolos-Pereira S, Callegaro D, Monteiro ML. Comparison of visual acuity and automated perimetry findings in patients with neuromyelitis optica or multiple sclerosis after single or multiple attacks of optic neuritis. J Neuroophthalmol. 2012;32(2):102-106.

18. Waters P, Jarius S, Littleton E, et al. Aquaporin-4 antibodies in neuromyelitis optica and longitudinally extensive transverse myelitis. Arch Neurol. 2008;65(7):913-919.

19. McDonald WI, Barnes D. The ocular manifestations of multiple sclerosis. 1. Abnormalities of the afferent visual system. J Neurol Neurosurg Psychiatry. 1992;55(9):747-752.

20. Slamovits TL, Rosen CE, Cheng KP, Striph GG. Visual recovery in patients with optic neuritis and visual loss to no light perception. Am J Ophthalmol. 1991;111(2):209-214.

21. Osuntokun BO. The pattern of neurological illness in tropical Africa. Experience at Ibadan, Nigeria. J Neurol Sci. 1971;12(4):417-442.

22. Kira J. Multiple sclerosis in the Japanese population. Lancet Neurol. 2003;2(2):117-127.

23. Naismith RT, Tutlam NT, Xu J, et al. Optical coherence tomography differs in neuromyelitis optica compared with multiple sclerosis. Neurology. 2009;72(12):1077-1082.

24. Merle H, Olindo S, Donnio A, Richer R, Smadja D, Cabre P. Retinal peripapillary nerve fiber layer thickness in neuromyelitis optica. Invest Ophthalmol Vis Sci. 2008;49(10):4412-4417.

25. Wang KC, Wang SJ, Lee CL, Chen SY, Tsai CP. The rescue effect of plasma exchange for neuromyelitis optica. J Clin Neurosci. 2011; 18(1):43-46.

26. Nakajima H, Hosokawa T, Sugino M, et al. Visual field defects of optic neuritis in neuromyelitis optica compared with multiple sclerosis. $B M C$ Neurol. 2010;10:45. 
27. Sadda SR, Nee M, Miller NR, Biousse V, Newman N, Kouzis A. Clinical spectrum of posterior ischemic optic neuropathy. Am J Ophthalmol 2001;132(5):743-750.

28. Mandler RN, Davis LE, Jeffery DR, Kornfeld M. Devic's neuromyelitis optica: a clinicopathological study of 8 patients. Ann Neurol. 1993;34(2): $162-168$.

29. Fardet L, Généreau T, Mikaeloff Y, Fontaine B, Seilhean D, Cabane J. Devic's neuromyelitis optica: study of nine cases. Acta Neurol Scand. 2003;108(3):193-200.
30. Green AJ, Cree BA. Distinctive retinal nerve fibre layer and vascular changes in neuromyelitis optica following optic neuritis. $J$ Neurol Neurosurg Psychiatry. 2009;80(9):1002-1005.

31. Roemer SF, Parisi JE, Lennon VA, et al. Pattern-specific loss of aquaporin-4 immunoreactivity distinguishes neuromyelitis optica from multiple sclerosis. Brain. 2007;130(Pt 5):1194-1205.

\section{Publish your work in this journal}

Clinical Ophthalmology is an international, peer-reviewed journal covering all subspecialties within ophthalmology. Key topics include: Optometry; Visual science; Pharmacology and drug therapy in eye diseases; Basic Sciences; Primary and Secondary eye care; Patien Safety and Quality of Care Improvements. This journal is indexed on

Submit your manuscript here: http://www.dovepress.com/clinical-ophthalmology-journal

\section{Dovepress}

PubMed Central and CAS, and is the official journal of The Society of Clinical Ophthalmology (SCO). The manuscript management system is completely online and includes a very quick and fair peer-review system, which is all easy to use. Visit http://www.dovepress.com/ testimonials.php to read real quotes from published authors. 